\title{
Consecuencias demográficas de la emigración y el retorno en Extremadura
}

\author{
BLANCA AZCÁRATE
}

\section{INTRODUCCION}

En Extremadura, donde la emigración a ultramar no ha tenido importancia, comienza a registrarse en la década de los cincuenta un movimiento de la población hacia las ya promocionadas regiones económicas de Madrid, Barcelona y provincias costeras vascas, donde el sector secundario y terciario sólo iba a poder absorber una parte de la mano de obra que se habia quedado sin trabajo. Esta importancia de la migración interior se refleja, sobre todo, en el freno del desarrollo económico que se detecta en la región extremeña.

Con la entrada en vigor del Plan de Estabilización el 21-7-1959, se instaura un proceso de crecimiento y modernización, en parte con ayuda de créditos extranjeros. Se comienza un proceso de mecanización de la agricultura - por ejemplo en zonas de regadío como La Vera-Jerte, donde se introduce la mecanización, con la consiguiente pérdida de puestos de trabajo-, lo que supondrá una disminución en el total de la población activa ocupada en este sector. Se va a producir entonces, a partir de finales de los cincuenta/principios de los sesenta, un movimiento de población hacia los países europeos industrializados, donde había un aumento de la demanda de mano de obra, consecuencia de un desarrollo económico favorable caracterizado por la reducción de las horas de trabajo y por una disminución de la población activa, debido sobre todo a una jubilación anticipada y a una entrada en la vida activa más tardia, constatándose, por tanto, una estrecha correlación entre las fases principales del desarrollo económico y determi- 
nadas corrientes y formas migratorias (cp. para el caso español MERTINS, 1986, pág. 42 s.).

Los países de destino preferentes en la emigración extremeña son Suiza, República Federal de Alemania (RFA) y Francia, destinos predominantes en todas las regiones españolas con fuerte emigración, si exceptuamos la emigración temporal a Francia de los andaluces. Mientras que en la RFA, desde 1960 , el volumen de la emigración extremeña (con el intervalo provocado por la recesión económica 1966/67) aumenta paulatinamente, se corta bruscamente desde 1973, debido a la suspensión de contratación de trabajadores extranjeros, decretado por el Gobierno germano en ese mismo año. En Suiza, caracterizada por el principio de rotación (HOFFMANN-NOWOTNY, 1973, pág. $41 \mathrm{ss}$.), se registra una parecida evolución hasta 1973, donde el corte, aunque existe, no es tan brusco como el registrado en la RFA.

En estos paises predomina una concentración de la población por nacionalidades en zonas concretas, como resultado de la atracción que produce el asentamiento en un determinado lugar por parte de habitantes de una región concreta, provocando además una reacción en cadena, y también a causa del interés de las empresas receptoras por obtener un máximo rendimiento de los alojamientos e instituciones creadas para el mejor aprovechamiento y aclimatación de los extranjeros (casas regionales, cursos de lengua, cursos específicos dentro de la empresa, escuelas... etc.) (cp. para el caso alemán: GIESE, 1978).

Entre las causas de la emigración actúan conjuntamente una serie de factores de atracción y de impulso. Así, aunque resulta muy difícil una atomización de estos factores, que nunca aparecen por aislado, intentaremos analizar estas causas estructurales de la emigración entendiéndolas como causas que actúan generalmente de una manera simultánea:

Las causas de estructura económica son las más relevantes y las que actúan como factor de impulso más evidente.

Extremadura es una región con una estructura agraria dualista que paraliza el desarrollo económico: frente a un gran número de pequeñas explotaciones, hay por otra parte pocas explotaciones grandes, que además representan una gran proporción de la superficie agrícola. El último censo agrario de 1982 parece constatar esta afirmación: 
- Las fincas mayores de $200 \mathrm{Ha}$. representan en Extremadura el 56,4 por 100 de la superficie agrícola utilizada, y las menores de $5 \mathrm{Ha}$. el 3,9 por 100 .

- El 56,3 por 100 de las explotaciones son menores de $5 \mathrm{Ha}$., y el 2,6 por 100 son mayores de $220 \mathrm{Ha}$.

Con la entrada de la mecanización, gran parte de la población campesina de las zonas latifundistas se vio inmersa en un subempleo latente, debido a esta modernización agraria que liberó mucha mano de obra agrícola, sin crear paralelamente puestos de trabajo en el sector secundario $y$ terciario. (SCHILLER, 1971; BERGER/HESSLER/KAVEMANN, 1970). En las áreas de regadío, tradicionalmente con alta densidad de población (La Vera-Jerte) y donde no es posible una ampliación del espacio agrícola, la mecanización también llevó consigo la pérdida de puestos de trabajo, actuando como factor importante en la decisión de emigrar, la presión creciente de población en relación con la cada vez más escasa oferta de puestos de trabajo.

También son importantes las causas derivadas de la estructura demográfica de las provincias extremeñas como son, entre otras, la existencia de unas altas tasas de natalidad -con el consiguiente alto crecimiento natural de la población (=presión de población), al no existir una evolución paralela de los recursos económicos-, o la falta de empleo en los sectores secundario y terciario, que queda reflejado en la proporción de población ocupada en los distintos sectores de actividad, con una proporción y dependencia altísima del sector primario en comparación con la media nacional (cp. Fig. 1). Además, se constatan unas tasas de paro cada vez más pronunciadas (tasas de paro en Extremadura: 1977, 8,4 \%; 1987, 26,4 \%).

En la figura 1, donde se representa la población activa distribuida según los distintos sectores de actividad para 1987 en Extremadura y en España, se observa con claridad la importancia que tiene todavía en esta región la población ocupada en el sector agrícola $(30,7 \%$ frente al $14,9 \%$ de España), y la ínfima relevancia del sector industrial ( $8,9 \%$ del total de activos frente al $24 \%$ nacional), fiel reflejo de la precaria industrialización de esta región y de su dependencia del sector agrícola. La importancia del sector primario en el PIB se reduce paulatinamente; sin embargo, la proporción tanto de España como de Extremadura es todavía muy alta. 
Ahora bien, junto a unas causas estructurales generales que explican y facilitan la decisión de emigrar, hay una serie de motivos personales, entre los que predominan los llamados motivos económicos:

a) la posibilidad de encontrar un puesto de trabajo;

b) o bien, de encontrar uno mejor remunerado, y así poder satisfacer deseos muy concretos, como puede ser la compra de una casa, la apertura de un negocio propio, el mantenimiento de la familia mediante envio de remesas, etc., casi todos ellos para demostrar la "elevación del status social» conseguido con el trabajo en el extranjero (cp. entre otros, LEIB, 1983; GERM, 1979).

Las consecuencias de los movimientos espaciales de la población son en general conocidas: 1) la reducción de la población, 2) la transformación de la estructura, y 3) los cambios producidos en el comportamiento natural de la población son las más importantes.

\section{REDUCCION GENERAL DE LA POBLACION}

En el período 1900/1986, la población de Extremadura ha registrado una evolución de los efectivos demográficos muy por debajo de la media nacional -123,8 por 100 de incremento, frente al 208,9 por 100 de España; cp. Fig. 2-. Esto se traduce en el hecho de que la importancia de la población extremeña en el total nacional tenga cada vez menos relevancia: en 1986 representaba solamente el 2,79 por 100 del total de la población española, es decir, casi la mitad de lo que representaba a comienzos de siglo (1900, 4,72\%; 1950, 4,85\%).

Ahora bien, en el desarrollo de la población durante el transcurso del presente siglo podemos, a grandes rasgos, diferenciar claramente dos etapas, aunque dentro de cada una de ellas encontremos oscilaciones y comportamientos diferenciados:

a) Hasta 1950 se registra un comportamiento en el desarrollo demográfico paralelo al acaecido a nivel nacional, con ligeras fluctuaciones; así se puede observar: 
- una primera fase, a comienzos de siglo, en la que el crecimiento de la población se acelera (en el periodo 1900/1910, el crecimiento intercensal de la población extremeña, supera incluso al crecimiento nacional);

- posteriormente, el desarrollo de la población va a ser más lento pero continuado, y con una evolución similar a la registrada a nivel nacional.

Los factores que pueden explicar este crecimiento continuo de la población hay que buscarlos, ante todo, en el comportamiento de la natalidad, con valores superiores a la media nacional en este período de tiempo, y con una mortalidad estacionaria, lo que se traduce en un alto crecimiento vegetativo.

b) Desde 1950 se va a registrar una regresión general de la población en las dos provincias de la región extremeña, aunque algo más acusada en la provincia de Cáceres (en el período 1950/1986 se reduce la población de Cáceres en un $22,8 \%$ y la de Badajoz en $18,5 \%$ ); y junto a la reducción general, una transformación de la estructura demográfica, que afectará al desarrollo del conjunto de la región extremeña.

Este corte brusco en el desarrollo de la población en la década de los 50 , viene marcado por el comienzo de los movimientos espaciales de la población, siendo la región extremeña una de las regiones de España que, junto con la región gallega y andaluza, más ha sufrido este proceso emigratorio, con una reducción de la población, motivada por estos movimientos (en 1986 se registra en ambas provincias menos población que la contabilizada en 1930 !!), pero también debido al predominio del carácter selectivo de la emigración.

Ya hemos apuntado que este descenso de la población afecta más profundamente a la provincia de Cáceres que a la de Badajoz. Ahora bien, dentro de éstas no se van a ver afectadas por igual las distintas comarcas que conforman cada provincia, detectándose, en líneas generales, una regresión más pronunciada en las comarcas de la provincia de Cáceres donde la totalidad de ellas, incluyendo sus cabeceras comarcales, registran disminución de los efectivos demográficos en el período 1950/1981, siendo para ambas provincias las áreas fronterizas las más afectadas - para la problemática de las áreas fronterizas, véase entre otros: CAMPESINO FERNÁN- 
DEZ, 1986; RUJAS LAZZARO, 1981; RAFFESTIN, 1974-. Se puede decir que sufren un mayor retroceso de población, aquellas comarcas que no poseen una cabecera comarcal importante, que actúe de foco de atracción de la población circundante.

En lo que respecta a la distribución espacial de la población, se puede observar a grandes rasgos, la concentración de la población en las capitales de provincia. No hay que olvidar el papel de los centros medianos en ambas provincias, como es el caso de Mérida en Badajoz, y de Plasencia en la provincia de Cáceres, que actúan como lugares centrales de un entorno de actividad agrícola en este último, y como centro gestor y administrativo en el primero.

Concentración motivada por movimientos interprovinciales e intraprovinciales hacia los núcleos mayores, y la consiguiente elevación de las tasas de natalidad, debido al predominio del carácter selectivo de la emigración, con inmigrantes entre 20 y 45 años, produciéndose paralelamente un proceso de despoblación de los espacios estructuralmente rurales. Estos contrastes entre concentración/despoblación son más acusados desde 1950.

Las transformaciones demográficas y socioeconómicas se reflejan en muchas ocasiones en la estructura espacial de la población. Cuanto más alta es la incidencia de la emigración en un asentamiento y más envejecida es la población de éste, más se detecta la transformación en el estado de las viviendas, bien por abandono o bien por derrumbamiento de las casas.

Esto es claramente visible en la región extremeña, donde una alta proporción de casas caídas y/o abandonadas siempre se correlaciona con una alta proporción envejecida, y en parte también con unas proporciones altas de mujeres y niños.

\section{TRANSFORMACION DE LA ESTRUCTURA DE LA POBLACION}

En el gráfico de la figura 3 se constatan diferentes fenómenos:

- La tendencia a disminuir proporcionalmente el grupo que representa a los menores de 15 años - aquella población que no es todavía económi- 
camente activa- - a lo largo del presente siglo, lo que predice una disminución en la evolución de las tasas de natalidad.

- Disminución de la población que denominamos adulta, potencialmente activa y reproductora, representada por el grupo de población que abarca desde los 15 a los 64 años, que al verse afectado repercute en la tasa de dependencia, con el carácter socioeconómico que conlleva, y lo que ello representa de lastre social para este grupo de población económicamente activo. Así tenemos una tasa de población económicamente activa con un bajo porcentaje $(44,3 \%$ en 1987$)$ en comparación con el total nacional $(49,1 \%$ en 1987$)$.

- Por último, el grupo de más de 65 años representa aquella parte de la población que no ejerce ya una actividad económica. Su tendencia hacia el aumento es evidente desde los años 50.

Con la proporción de población en este grupo de edad, queda reflejado el índice de envejecimiento de Extremadura, y podemos observar el progresivo envejecimiento de ésta $(1940,5,7 \% ; 1950,7,6 \% ; 1981,13,4 \%)$.

\section{TRANSFORMACION DEL COMPORTAMIENTO NATURAL}

Las consecuencias demográficas en el comportamiento natural de la población, son también notables. El efecto selectivo de la emigración, es decir, la emigración de jóvenes activos, mejor formados y cualificados, la mayoría de las veces provoca en las áreas de origen fenómenos que enumeramos a continuación:

a) Un retroceso de la tasa de natalidad, que depende en gran medida de la composición por edades de la población. En 1984 los valores de la tasa de natalidad en la provincia de Cáceres son más bajos que la media nacional, pero no ha sido así siempre, pues durante el presente siglo y hasta principios de los años sesenta, la natalidad ha estado siempre por encima de la media nacional (provincia de Cáceres: 1930, 3,5 \%; 1960, 2,4 \%; 1984, 1,1 \% / España: 1930, $2,8 \% ; 1960,2,1 \% ; 1984,1,2 \%$ ). En la provincia de Badajoz, la tasa de natalidad está por encima de la media nacional durante la 
primera mitad del siglo xx, sufriendo una fuerte reducción en la década de los sesenta, pero recuperándose a partir de comienzos de los ochenta (1930, 2,9\%; 1960, 2,1\%; 1984, 1,4\%).

Para explicar esta reducción de la natalidad, hemos de recurrir en mayor medida a la pérdida de población potencialmente reproductora, es decir, a los cambios sufridos en la estructura de edades y sexo de la población, sin olvidar que para analizar el comportamiento de la natalidad de una determinada sociedad hay que considerar el medio sociocultural en el que se desarrolla.

b) La mortalidad registra similares comportamientos en su evolución, que corresponden a sociedades desarrolladas, con una reducción continuada a lo largo del siglo Xx, sobre todo de la mortalidad infantil, así como un aumento paulatino de la esperanza de vida. A pesar de todo, los valores de la tasa de mortalidad siguen siendo en 1984 mayores que la media nacional (1984: Cáceres, 0,90\%; Badajoz, 0,95\% y España, 0,77\%).

Desde principios de los años ochenta se detecta en algunos municipios un cambio de signo en la evolución de la población, registrándose en algunos casos una estabilización e incluso un ligero crecimiento. Esto se debe sobre todo al freno del proceso emigratorio y en algunas áreas a un incipiente retorno de la población emigrada.

En las zonas afectadas predominantemente por movimientos migratorios interiores, el movimiento de retorno es menos significativo, pues, por regla general, éstos tienen una ruptura más fuerte con el municipio de origen.

Siguiendo el estudio realizado por RHOADES (1978), podemos afirmar que los factores que determinan el lugar definitivo de asentamiento a la hora del retorno son la edad, el estado civil y los ahorros conseguidos durante su estancia en el extranjero, este último factor está en relación directa con los años permanecidos fuera. Así, distingue:

a) Jóvenes solteros, que retornan a su municipio de origen en un primer momento, para posteriormente trasladarse hacia un centro económico supra-regional, dentro del ámbito nacional. 
b) Jóvenes y casados retornan preferentemente a capitales de provincia, obteniendo así una mejor oportunidad de encontrar puestos de trabajo en el sector industrial o de servicios.

c) Adultos y casados, regresan con más frecuencia a su lugar de origen, o al núcleo comarcal cercano más importante, cuando aquél no ofrezca la infraestructura suficiente al haber estado más afectado por las consecuencias sufridas por la emigración (abandono, envejecimiento externo, etc.).

Este proceso en la elección del lugar de retorno provoca una redistribución interior de la población que conlleva una agudización de las disparidades regionales tanto en la estructura de la población, como en la estructura económica.

Las consecuencias de la emigración y el retorno en las áreas de origen están determinadas por el tipo de emigración, así como de la distinta utilización que realicen de sus ahorros y el comportamiento en la inversión de los emigrantes y/o retornados. Está demostrado en algunas investigaciones realizadas en las áreas de origen no sólo de la región extremeña, sino también en otras áreas españolas con fuerte emigración, como Galicia y Andalucía (cp. AZCÁRATE/MERTINS, 1984; GROTTENDIECK, 1984), que son múltiples las causas que influyen en el volumen y repercusiones de la emigración, como son los factores naturales, económicos, demográficos, etc. Asimismo, la duración de la estancia en el extranjero y la mayor o menor relación con el lugar de origen durante la emigración, entre otros factores, van a influir posteriormente en la redistribución espacial de la población retornada. 


\section{BIBLIOGRAFIA}

AzCÁRATE, B.: Ursachen, Umfang und Auswirkungen der Abwanderung im ländlichen Bereich Galiciens am Beispiel der Provinz Orense. Berna - Frankurt - Nueva York 1985.

AzCÁRATE, B.; MeRtins, G.: «Determinanten und Auswirkungen der Arbeitsmigration auf die Bevölkerungs-, Siedlungs- und Wirtschaftsstruktur im ländlichen Raum Galiciens. Fallbeispiele aus der Provinz Orense". En: MERTINS (Ed.): Untersuchungen zur spanischen Arbeitsmigration. Marburger Geographische Schriften, núm. 5, Marburg/Lahn 1984, pp. 1-56.

BALABANIAN, O.: «Les explotations et les problemes de l'agriculture en Estremadure Espagnole et dans le Haut-Alentejo". Contribution a l'étude du campagnes mediterraneennes 1980 .

BARRIENTOS Alfageme, G.: El envejecimiento: un problema estructural de la población cacereña. Cáceres 1981.

BeRger, H./Hessler, M./Kavemann, B.: Brot für heute, Hunger für morgen. Landarbeiter in Südspanien. Frankfurt/Main 1978.

Campesino Fernández, J. A.: La frontera como factor geográfico. Situación actual de la investigación peninsular. Primeras Jornadas Ibéricas de investigadores en Ciencias humanas y sociales. Olivenza 1985.

-: "Las Comarcas fronterizas extremeñas: La otra cara simétrica de la Miseria». En: Estudos em homenagem a Mariano Feio. Évora 1986.

Castillo y Castillo, J.: La emigración española en la encrucijada. Estudio empírico de la emigración de retorno. Madrid 1980. 
CAzorla Pérez, J.: Emigración y retorno. Una perspectiva europea. Madrid 1981.

Garcia Zarza, E.: «Evolución, estructura y otros aspectos de la población cacereña». En: Revista de Estudios Extremeños, t. XXXIII, núm. 1 Badajoz 1977.

Germ (Grupo de Estudios sobre Reintegración de Migrantes): Fremd im eigenen Land. Materialien und Interviews zur Rückkehr spanischer Migranten. Madrid 1979.

GIESE, E.: «Räumliche Difussion ausländischer Arbeitnehmer in der Bundesrepublik Deutschland 1960-1976». En: Die Erde 109, Berlin 1978, pp. 92-110.

GrotTENDIECK, J.: “Determinanten und Auswirkungen der Arbeitsmigration wie -remigration in Latifudiengebieten Ostandalusiens". En MERTINS (Ed.): Untersuchungen zur spanischen Arbeitsmigration. Marburger Geographische Schriften, núm. 5, Marburg/Lahn 1984, pp. 57-102.

Hoffmann-Nowotny, H. J.: Migration. Ein Beitrag zu einer soziologischen Erklärung. Stuttgart 1970.

KADE, G., SCHILLER, G.: "Los trabajadores andaluces en Alemania. Resultados de una investigación". En: Anales de Sociología, 4/5, Barcelona 1968/1969, pp. 159-188.

KNUTH, B.: «Binnenwanderung, Emigration un Remigration in der Extremadura». En: MERTINS (Ed.): Untersuchungen zur spanischen Arbeitsmigration. Marburger Geographische Schriften, núm. 5. Marburg/Lahn 1984, pp. 103-150.

LEIB, J.: “Rimessen, Ersparnisverwendung und Investitionsverhalten. Das Beispiel Spanien." En: Geographische Rundschau, 35, Braunschweig 1983, pp. 54-60.

MERTINS, G.: «Rückwanderung spanischer Arbeitnehmer aus dem europäischen Ausland. Räumliches Verteilungsmuster und Investitionsverhalten in Spanien». En: KÖRNER, H.; M. WERTH (Eds.): Rückwanderung und Reintegration von ausländischen 
Arbeitnehmern in Europa. Schriften des Instituts für Entwicklungsforschung. Wirtschafts und Sozialplanung, t. 1. Saarbrücken 1981, pp. 63-75.

RAFFESTIN, $\mathrm{Cl}$.: “Élements pour une problématique des régions frontaliéres». En: L'Espace Géographique, núm. 1, 1974,. pp. 1218.

RHOADES, R.: Intra-European return migration and rural development. Lessons from the spanish case 1978.

RUJAS LAZARO, M.: «El desarrollo de las regiones mediante la cooperación trans-fronteriza». En: Revista de Estudios Territoriales, 1, 1981, pp. 127-133. Madrid, CEOTMA.

SCHILLER, G.: Europäische Arbeitskräftemobilität und wirtschaftliche Entwicklung der Mittelmeerländer. Eine empirische Untersuchung über die Wirkungen der Gastarbeiterwanderungen auf die Abgabeländer. Darmstadt 1971.

SIGUÁN SOLER, M.: «La emigración y su influencia sobre el desarrollo del campo". En: Revista de Estudios Agro-sociales, 58, Madrid 1967, pp. 49-71. 


\section{FIGURA 1. OCUPADOS POR SECTORES DE ACTIVIDAD (1987)}
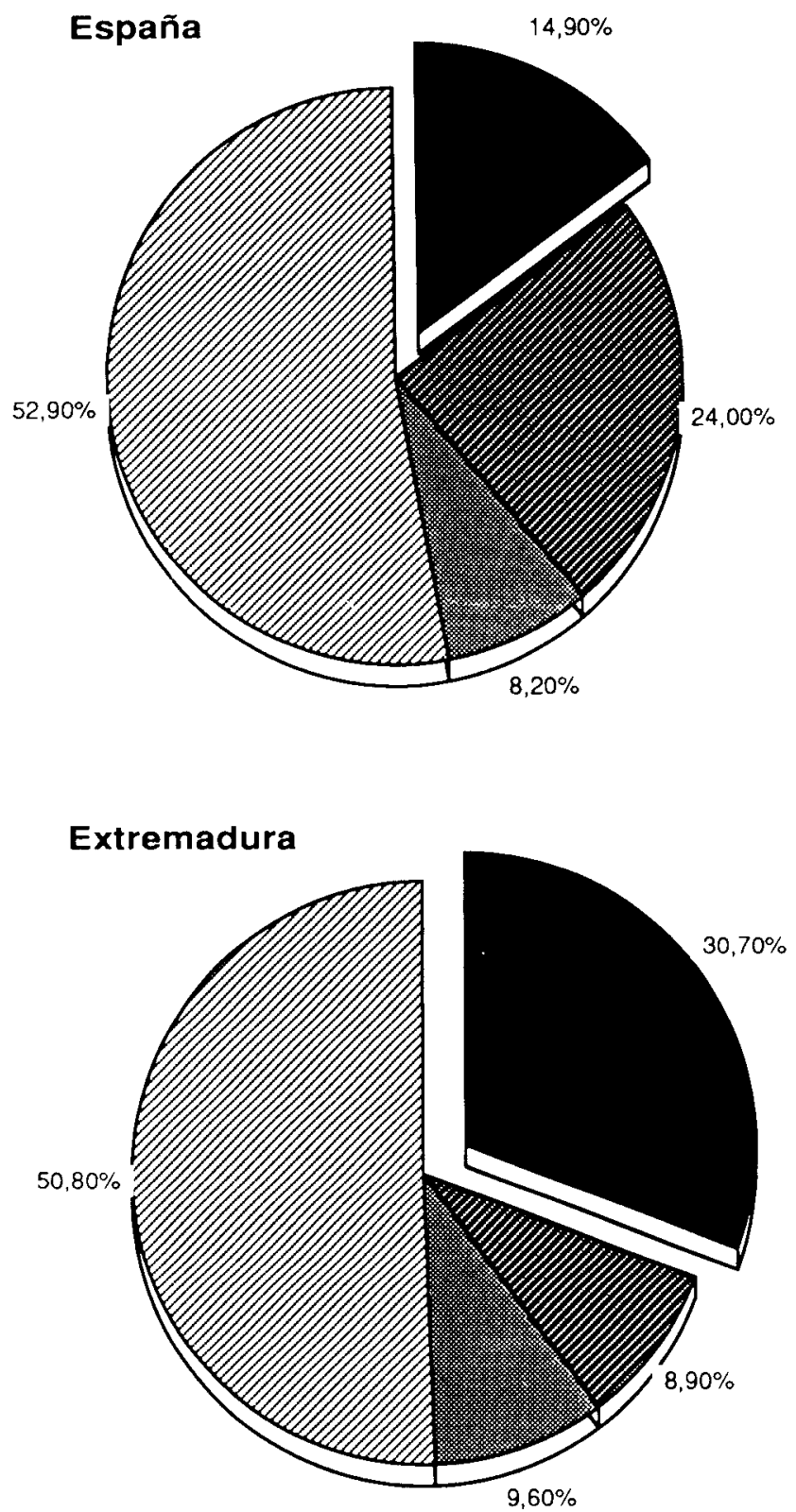

(c) B. Azcárate 1988 


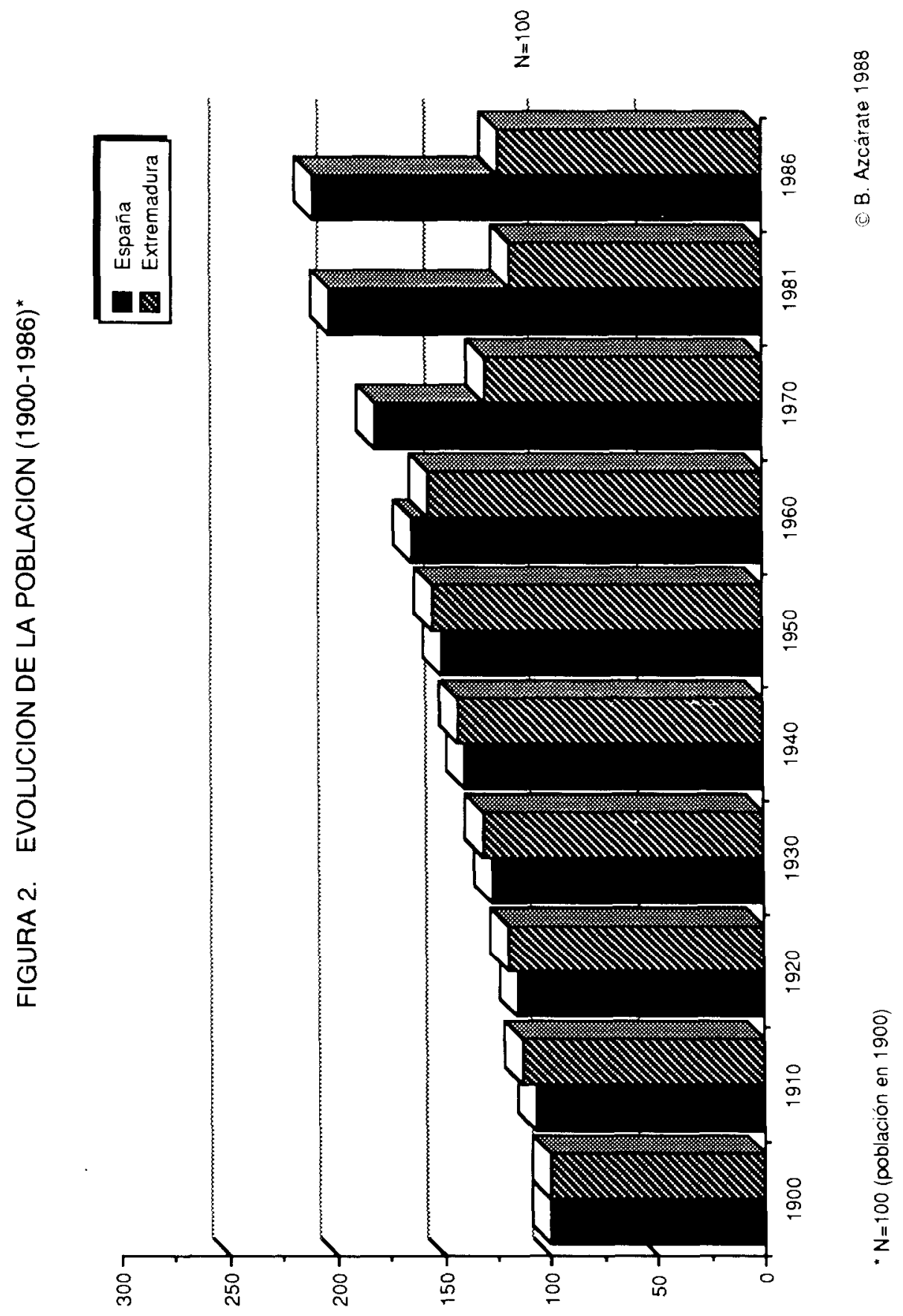




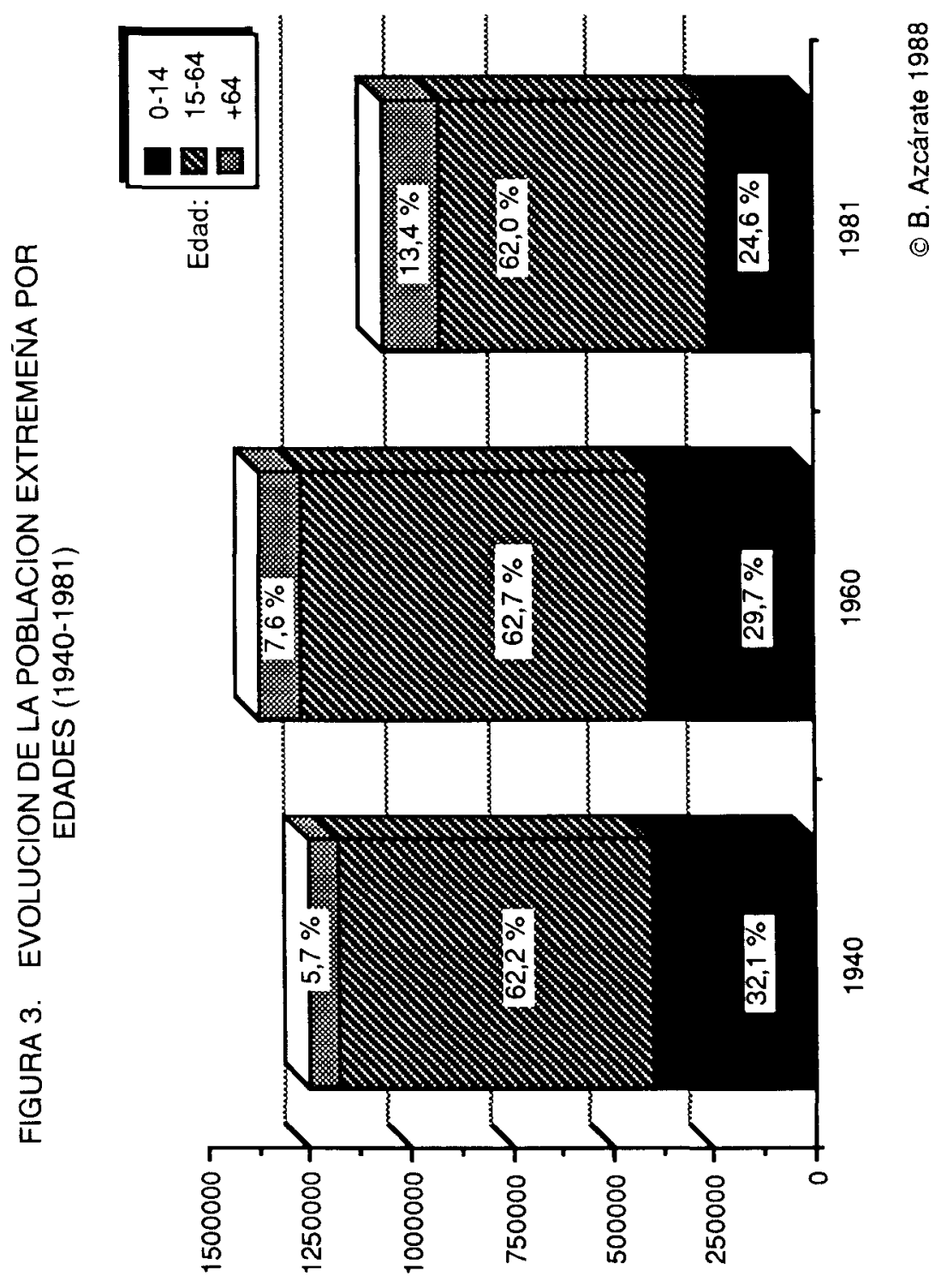

sejueflqey op odewnN 